\section{A pediatric case of Meckel diverticulum with uncommon presentation showing no lower gastrointestinal bleeding}

\author{
Sanaz Mehrabani, Soheil Osia \\ Non-Communicable Pediatric Diseases \\ Research Center, Health Research \\ Institute, Babol University of Medical \\ Sciences, Iran
}

\begin{abstract}
Meckel's diverticulum (MD) is the most common congenital abnormality of the gastrointestinal tract. Painless intestinal hemorrhage is a frequently occurring complication that relates to MD in children. Bowel obstruction is a rare complication of MD in children. We report on the case of a fouryear old male child who presented with abdominal pain and hematemesis. Finally, bowel obstruction due to MD was confirmed at surgery.
\end{abstract}

\section{Introduction}

Meckel's diverticulum (MD), the most common congenital anomaly of the gastrointestinal tract, has been described as the remnant of the omphalomesenteric duct. ${ }^{1}$ According to the rule of $2 \mathrm{~s}, \mathrm{MD}$ occurs in $2 \%$ of the population, with a male to female ratio of $2: 1$, is located 2 feet proximal to the ileocecal valve, the symptoms present $\leq 2.0$ years, and it is approximately 2 inches long. 1,2

The prevalence of MD depends on the population studied. It increases, in descending order, in children with major malformation of the umbilicus, alimentary tract, nervous system, or cardiovascular system. ${ }^{3}$

Meckel's diverticulum is often clinically silent, particularly in adults, and the symptomatic picture may be abdominal pain, bowel obstruction or gastrointestinal bleeding. ${ }^{4}$ We describe the case a four-yearold child who presented with intestinal volvulus of the MD, an uncommon phenomenon in children of this age.

\section{Case Report}

A four-year-old male child was admitted to the emergency department of Amirkola Children's Hospital, Mazandran, Iran, with complaints of abdominal pain. It was associated with oral intolerance and recurrent vomiting. The child vomited blood twice on the same day. There was no history of fever, diarrhea and bleeding per rectum, with normal defecation. On physical examination, his vital signs were within the normal limits (blood pressure of 90/50 $\mathrm{mm} / \mathrm{Hg}$, a respiratory rate of 24 breaths/minute, a pulse rate of 90 beats/minute, and a temperature of $36.5^{\circ} \mathrm{C}$ ), without dehydration.

On abdominal examination, pain was located in the periumbilical and epigastric area, but the abdomen was soft without tenderness and organomegaly. No masses were felt and the bowel sounds were normal.

A nasogastric tube was inserted and washing performed with $300 \mathrm{cc}$ normal saline for the bloody vomiting, resulting in the extraction of a coffee ground-like discharge and blood clots. An endoscopy was carried out owing to the hematemesis. Prolapse gastropathy and subepithelial hemorrhage of the gastric fundus was determined.

The abdomen became mildly distended with diffuse tenderness on palpation on the second day and the pain shifted to the right upper quadrant.

The first biochemical measurement showed leukocytosis with neutrophilia, thrombocytosis, and positive ketones in the urinalysis test. The rest of the laboratory data were within the normal limits.

An erect abdominal X-ray revealed air fluid levels (Figure 1). Free fluid in the left and right paracolic gutters and Morison's pouch were demonstrated on ultrasound of the abdomen. Due to the acute abdominal picture on the second day, emergency surgery counseling was carried out, and based on the recommendation of the pediatric surgeon, the child was taken to the operating room. An abdominal computed tomography (CT) scan could not be performed because of the emergency nature of the situation. Surgical evaluation under general anesthesia was performed, and a transverse laparotomy incision was made. MD was found 30 $\mathrm{cm}$ proximal to the ileocecal valve (Figure 2). Pelvic peritoneal adhesions were observed. The small intestines were distended, without perforation and necrosis, but had twisted around the MD, causing obstruction and volvulus. The MD was delivered outside of the abdomen. Adhesion without distinctive cause had affected the proximal, distal limb of the small intestine. Thus, resection of the involved section with primary anastomosis and appendectomy was carried out. After eight days of surgery, the patient was discharged without complications. MD without ectopic tissue was revealed on histology.
Correspondence: Sanaz Mehrabani, NonCommunicable Pediatric Diseases Research Center, No 19, Amirkola Children's Hospital, Amirkola, Babol, Mazandaran Province, 47317 41151 IR, Iran.

Tel.: +98.911.2511184 - Fax: +98.11.32346963.

E-mail: mehrabanisanaz@gmail.com

Key words: Gastrointestinal hemorrage, Volvolus, Meckel Diverticulum, Intestinal obstruction.

Acknowledgements: the authors are grateful to all the participants in this study for their cooperation.

Contributions: MS drafted the initial manuscript; MS and OS reviewed and revised the manuscript and approved the final manuscript as submitted.

Conflict of interest: the authors declare no potential conflict of interest.

Received for publication: 16 November 2016 .

Revision received: 1 February 2017.

Accepted for publication: 2 February 2017.

This work is licensed under a Creative Commons Attribution NonCommercial 4.0 License (CC BY-NC 4.0).

(C) Copyright S. Mehrabani and S. Osia, 2017 Licensee PAGEPress, Italy

Pediatric Reports 2017; 9:6973

doi:10.4081/pr.2017.6973

\section{Discussion}

Although most MD is asymptomatic, if complications such as hemorrhage, perforation, intestinal obstruction, and diverticulitis occur, symptoms can appear in patients with MD. More than half of the patients who develop symptoms are aged $\leq 2$ years. ${ }^{5}$

Meckel's diverticulum often presents with hemorrhage or painless intestinal bleeding in children, in contrast to the symptoms of small bowel obstruction in adults. ${ }^{6} \mathrm{MD}$ is the most common reason for massive lower gastrointestinal tract bleeding that is painless in children, or for massive melena, leading to shock. ${ }^{7}$ However, it should be born in mind that the presentation of MD is non-specific and can be variable. 6

An intestinal obstruction relating to $\mathrm{MD}$ in children can result from intussusception, volvulus, diverticulitis, and Littre hernia. ${ }^{8}$ ENREF_9 Of these, intussusception with $\mathrm{MD}$ as the leading point, followed by a mechanical volvulus of the small intestine around a persistent band that has attached the MD to the umbilicus, ${ }^{8}$ ENREF_10 are the most common. 
Younger children with symptomatic MD are more susceptible to presenting with obstructive symptoms. ${ }^{9}$ The pediatric population in whom there is obstruction may display irritability, abdominal distension, abdominal pain, anorexia, nausea, and vomiting. 8 ENREF 9

Half of MD-associated intussusception occurs in patients aged $\geq 3$ years. ${ }^{7}$ Volvulus results when the small intestine twists around a fibrous cord, or Meckel's band, tethering the MD to the umbilicus. ${ }^{7}$ It was shown in a case series study on 34 children with MD that the most common chief complaint was lower gastrointestinal bleeding (35.3\%), abdominal pain (32.4\%), vomiting $(8.9 \%)$, and abdominal distension $(2.9 \%) .{ }^{8}$ The most common MD symptoms in children in the study by Lee et al. ${ }^{10}$ were lower gastrointestinal bleeding (47.0\%) and intestinal obstruction (32.4\%). Our case is uncommon because the child first presented with upper gastrointestinal bleeding, as opposed to the more usual lower gastrointestinal bleeding, followed by bowel obstruction. ${ }^{6}$

Neither intestinal obstruction nor volvulus due to MD are common features in chil- dren aged $\geq 2$ years. ${ }^{6}$ In addition, the reports on pediatric cases of intestinal volvulus due to MD are few in number. ${ }^{11}$ ENREF_14

The imaging investigation did not support a valid finding of MD and volvulus in our study as a CT scan was not performed because this was an emergency, for which surgery was urgently required. Despite their low specificity, the use of radiological or sonographic modalities is clinically preferred to a Meckel's diverticulum scan for children with MD-associated obstructive symptoms since MD accounts for only a small fraction of the intestinal obstruction. Although some imaging clues might suggest MD and can be determined by an experienced radiologist, a routine $\mathrm{CT}$ scan is not advocated in patients presenting with intestinal obstruction. ${ }^{12}$ ENREF 15

Bloody vomiting and abdominal pain, as a primary presentation in children, are indicative of several diagnoses. However, if bilious vomiting occurs first, then it is more probable that it is bowel obstruction. In our case, the patient presented with generalized abdominal pain, with the bowel obstruction developing gradually over a 48 -hour period from admission.
Prolapse gastropathy is thought to occur after retching and forceful emesis. The gastric mucosa prolapses into the lower esophageal sphincter. Direct trauma to the mucosa occurs when the gastric mucosa becomes incarcerated through the lower esophageal sphincter. ${ }^{13}$ This may cause submucosal hemorrhage and superficial ulceration. ${ }^{14}$ Biopsy of the affected mucosa often shows mucosal inflammation. ${ }^{15}$ The most common area involved is the stomach fundus to the left of the esophageal lumen. Treatment was achieved by controlling the primary cause of vomiting in our patient. So, prolapse gastropathy is a nonspecific finding that there was in our patient from the beginning of symptoms.

\section{Conclusions}

Meckel's diverticulum should be considered in the differential diagnosis of acute abdominal pain and the signs and symptoms of bowel obstruction.

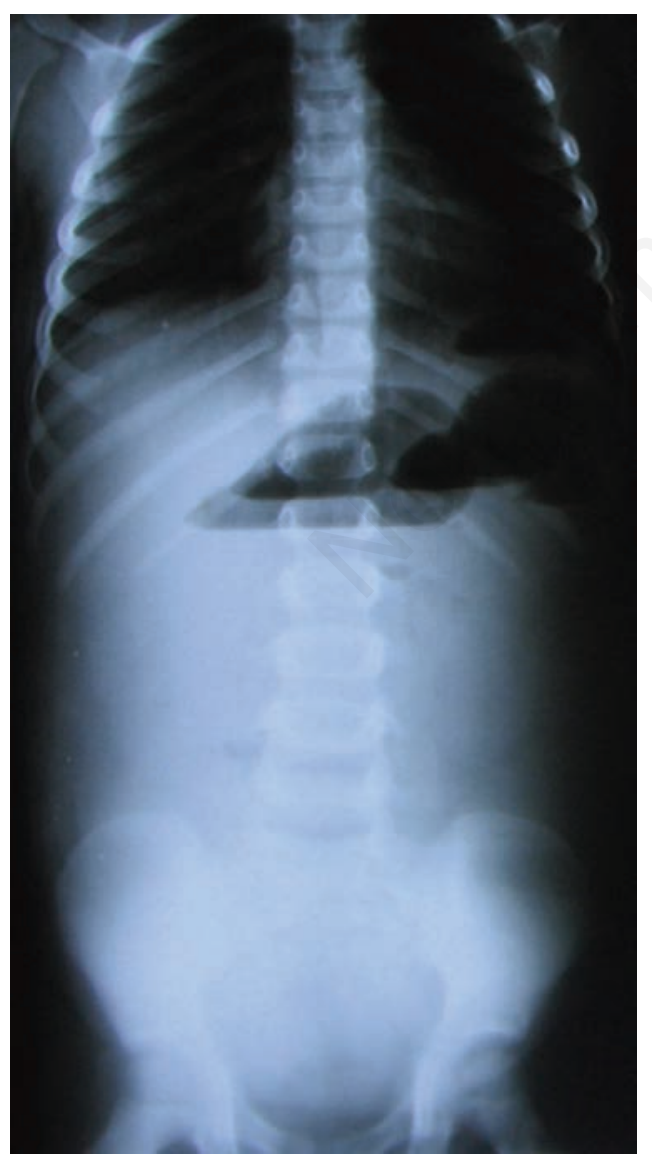

Figure 1. Supine abdominal plain film.

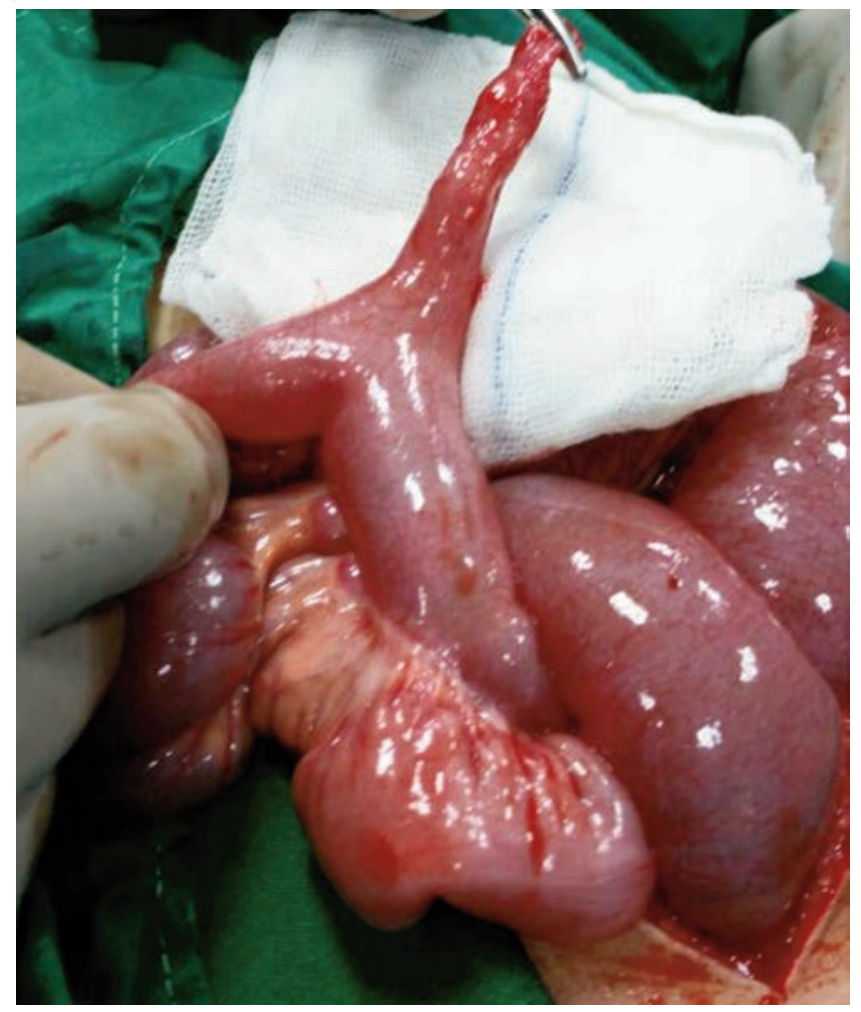

Figure 2. Intraoperative photograph showing portion of bowel obstruction due to Meckel's diverticulum ischemic bowel. 


\section{References}

1. Tenreiro N, Moreira H, Silva S, et al. Unusual presentation of a Meckel's diverticulum: a case report. J Surg Case Rep 2015;16:48-51.

2. Yahchouchy EK, Marano AF, Etienne JCF, Fingerhut AL. Meckel's diverticulum. J Am Coll Surg 2001;192:658-62.

3. Chen JJ, Lee HC, Yeung CY, et al. Meckel's diverticulum: factors associated with clinical manifestations. ISRN Gastroenterol 2014;2014.

4. Novoa RA, Shaffer K. Meckel's diverticulitis presenting with abdominal pain and angina. Case Rep Radiol 2008;3: 166.

5. Malhotra S, Roth DA, Gouge TH, et al. Gangrene of Meckel's diverticulum secondary to axial torsion: a rare complication. Am J Gastroenterol 1998;93:13735.
6. Javid P, Pauli EM. Meckel's diverticulum. 2016. Available from: http://www.uptodate.com/contents/mec $\mathrm{kels}$ - diverticulum? s our ce = search_result\&search $=$ Meckel $\% 27 \mathrm{~s}+\mathrm{di}$ verticulum\&selectedTitle $=1 \% 7 \mathrm{E} 34$

7. Huang CC, Lai MW, Hwang FM, et al. Diverse presentations in pediatric Meckel's diverticulum: a review of 100 cases. Pediatr Neonatol 2014;55:36975.

8. Blevrakis E, Partalis N, Seremeti C, Sakellaris G. Meckel's diverticulum in paediatric practice on Crete: a 10-year review. Afr J Paediatr Surg 2011;8:279.

9. Tseng YY, Yang YJ. Clinical and diagnostic relevance of Meckel's diverticulum in children. Eur J Pediatr 2009; 168:1519-23.

10. Lee YA, Seo JH, Youn HS, et al. Clinical features of symptomatic meckel's diverticulum. Korean J Pediatr
Gastroenterol Nutr 2006;9:193-9.

11. Shah B, Degloorkar S, Rao A. Volvulus of Meckel's diveticulum: a rare cause of intestinal obstruction in children. JCR 2015;5:121-3.

12. Olson DE, Kim YW, Donnelly LF. CT findings in children with Meckel diverticulum. Pediatr Radiol 2009;39:65963.

13. Pohl JF, Melin-Aldana H, Rudolph C. Prolapse gastropathy in the pediatric patients. J Pediatr Gastroenterol Nutr 2000;30:458-60.

14. Bishop PR, Nowicki MJ, Parker PH. Vomiting-induced hematemesis in children: Mallory-Weiss tear or prolapse gastropathy? J Pediatr Gastroenterol Nutr 2000;30:436-41.

15. Thomas E, Khatak KG. Hemorrhage due to retrograde prolapse of stomach. Am J Gastroenterology 1979;71:47780. 